\title{
A SOCIAL WELFARE ESTIMATION OF RIDE-SHARING IN CHINA: EVIDENCE FROM TRANSACTION DATA ANALYSIS OF A LARGE ONLINE PLATFORM
}

\author{
Benxi WANG ${ }^{1}$, Yi SHAO ${ }^{2}$, Meng $\mathrm{MIAO}^{3 *}$ \\ ${ }^{1,3}$ Renmin University of China, Beijing, China \\ ${ }^{2}$ Peking University, Beijing China
}

Received 25 April 2021; accepted 08 October 2021; first published online 02 February 2022

\begin{abstract}
This paper estimates the social welfare effect of China's largest online ride-sharing platform. Under the plausible assumption that consumers would change from traditional transportation to online ride-sharing when the marginal benefit of saved time outgrows the additional cost, we calculate the distribution of implied wage rate of passengers. We then use the passenger wage rate to calculate the social welfare generated by the decrease in waiting time and the reduction of waiting uncertainty brought about by the ride-sharing platform. Our estimate suggests that the ride-sharing platform created a total of 130.5 billion Yuan of social welfare in the three years between 2016 and 2018, and the consumer surplus and producer surplus created by an average transaction are 5.4 Yuan and 2.5 Yuan, respectively. The robustness test finds that our results were insensitive to the assumed risk aversion coefficient in the model, the subsample number used for each city, and the inclusion of nonlinear terms in the model. Alternative hypotheses, such as learning effect, seem unable to explain our result.
\end{abstract}

Keywords: social welfare, online ride-sharing platform, regulation.

JEL Classification: I30, I31, I38.

\section{Introduction}

Recently, the online car-hailing business has expanded rapidly, but its utility remains controversial. The supporter would say that, by optimally matching the passengers' demand and drivers' supply through the sharing mechanism and big data matching algorithm, the ridesharing platforms reduce transaction costs, reduce waiting time, and improve efficiency and social welfare. The opponent, however, believes that the rapid development of the online carhailing industry has also brought a series of other social problems, e.g., safety issues and the occupation of transportation resources such as roads (Shen \& Su, 2017). Also, it is believed that online car-hailing may have a negative impact on the livelihood of people in certain

*Corresponding author. E-mail: miaomeng@ruc.edu.cn

Copyright (c) 2022 The Author(s). Published by Vilnius Gediminas Technical University

This is an Open Access article distributed under the terms of the Creative Commons Attribution License (http://creativecommons. org/licenses/by/4.0/), which permits unrestricted use, distribution, and reproduction in any medium, provided the original author and source are credited. 
professions, such as taxi drivers. Therefore, there has been incessant call for the regulation of online car-hailing.

The decision as to whether to regulate ride-hailing should be based on correct quantification of the social welfare created by the ride-hailing business. Social welfare is referred to as the sum of the driver and passenger surplus, or the difference between the transaction price and the reserved prices. Despite numerous estimations of the social welfare created by ride-hailing platforms in developed countries, there are few studies trying to quantify the effect in these different places, where different social-economic statuses may lead to different conclusions. Moreover, the literature seems to be silent on the impact of regulation on social welfare creation by ride-hailing platforms.

In this paper, we try to address these two issues by an event study of China. China has the largest ride-hailing market in the developing world, and it has grown rapidly in recent years. In 2018, ride-sharing market transactions reached 247.8 billion Yuan. In the same year, the number of users in the ride-sharing market reached 333 million, 40.9 percent up from 2017. The ride-sharing platform carried about 20 billion passengers, accounting for $36.3 \%$ of the total number of taxi passengers. The penetration rate of ride-hailing users among netizens reached 43.2 percent, an increase of 26.2 percent in 2015 . At the same time, China also has very strict regulations on online ride-hailing. In 2018, two young ladies were killed by ride-hailing drivers in two isolated cases. The Chinese government took the opportunity to advocate for and implement very strict regulation on ride-hailing, despite the fact that the homicide rate of ride-hailing statistically is merely one-thirteenth of that of ordinary taxis. Transport authorities across the country have started to impose very high entry barriers as well, such as examinations for drivers and displacement limits for vehicles.

To estimate the social welfare created by ride-hailing and the impact of regulation, we make use of a highly unique dataset coming from the largest national ride-hailing platform in China. We call it "The Platform", or "TP" herein. As one of the earliest and leading companies in the industry, this company started its ride-hailing business in collaboration with taxis back in 2012 and launched its own ride-hailing vehicles in 2015. The total number of TP orders accounts for more than 80 percent of the country's total ride-hailing orders. We used transaction data of the TP orders in 359 major cities across the country over a threeyear period, from 2016 to 2018, to estimate producer and consumer surplus, respectively. To the best of our knowledge, our data is the largest study of its kind.

We use an innovative method to estimate social welfare. The basic information is the date when passengers enter the ride-hailing market. The assumption is that passengers, when deciding whether to enter the market or not, would fully consider the benefit (i.e., shorter time, less uncertainty) and disadvantages (higher prices) of ride-hailing compared to other traditional transportation and would only enter if the benefit outweighed the disadvantages. It is plausible to expect that those passengers with higher wage rates are entering the market earlier, as the times saved by car-hailing are associated with higher monetary value. We therefore calculated the implied passengers' wage rate using the difference in transportation time, uncertainty and cost between ride-hailing and other traditional means of transportation at the time of entry. Then, the wage rate of passengers is used to estimate the social welfare generated by the reduction of waiting time and the reduction of waiting uncertainty brought 
about by the increase in scale effect of online ride-hailing passengers after entering. We did a similar calculation for the drivers.

Our results prove that the existence of ride-hailing provides great social benefit. The ride-hailing platform has created a total of 130.5 billion Yuan of social welfare in the three years from 2016 to 18 , saving passengers more than 1.7 billion hours of commuting time. The consumer surplus and producer surplus created by a single order are 5.4 Yuan and 2.5 Yuan, respectively. After obtaining the baseline estimate results, we conducted a series of robustness tests. We find that our result is almost unchanged when we change the hypothesized people's risk aversion degree and customer base segmentation method. The result is robust to the introduction of the non-linear effect in the model and the consideration of shared bicycles and learning effect of passengers.

At the same time, we found that the regulation had a huge negative impact on the social welfare created by ride-hailing services. The implementation of the regulatory policies in 2018 resulted in a sharp contraction of the market size of online ride-hailing. This corresponds to a reduction of about 20 billion Yuan in social benefits. This is greater than the total passengers' welfare created by Uber in United States in 2015.

Our paper joins the literature on the social benefits of shared travel. Many scholars have long since started to estimate the social welfare impact of ride-sharing platforms. Cohen et al. (2016) used the data of Uber, the largest online car-hailing platform in the United States, to calculate the social welfare of ride-sharing. They used the mark-up behavior of passengers in different road scenarios to infer their willingness to pay and their social welfare. They concluded that Uber generated $\$ 6.8$ billion in social benefits in 2015 , with an average of $\$ 1.6$ per dollar transaction. The estimation method of Cohen et al. (2016) is widely considered to overestimate the social welfare of ride-sharing platforms. After considering the substitution effect between different modes of travel, Lam and Liu (2017) found that every dollar of transaction can create 72 cents of social welfare, a much lower figure. A result of similar result is achieved by Castillo (2020). Other studies have also fully explored a series of ways that ridesharing platforms can create social welfare, including increasing work flexibility (Hall et al., 2017; Hall \& Krueger, 2018; Chen et al., 2019; Li et al., 2021), enhancing ridership (Sadowsky \& Nelson, 2107; Hall et al., 2018; Grahn et al., 2021), reducing drunk driving (Greenwood \& Wattal, 2017; Athey \& Luca, 2019), increasing entrepreneurship and economists employment (Burtch et al., 2016), promoting local consumption (Zhang \& Li, 2017), reducing sexual harassment and bias (Park et al., 2017; Ge et al., 2020; Mejia \& Parker, 2021), reducing private car ownership (Gong et al., 2017), reducing information cost and road congestion (Shapiro, 2018; Goldfarb \& Tucker, 2019; Moskatel \& Slusky, 2019; Liu et al., 2021), etc. Certain negative evidence is also suggested by the literature, such as decreasing of incumbent taxi drivers' income and that of the entering ride-hailing drivers (Berger et al., 2018; Zoepf et al., 2018). See Yu et al. (2018) for a literature review. Our estimate of social welfare is closest to that of Lam and Liu (2017). At the same time, our paper also supplements the literature with the discussion of the reduction of social welfare caused by regulation.

The rest of our paper is arranged as follows: Section 1 describes the model and method of online car-hailing welfare estimation, Section 2 gives the sample data description and empirical analysis results, Section 3 offers the more detailed robustness test results, and the last Section summarizes the whole article and discusses the relevant policy implications. 


\section{Model and of social welfare estimation method}

\subsection{Model setting}

We firstly estimate the surplus obtained by passengers. Our estimate relies on two basic assumptions. First of all, passengers' travel decisions and travel method decisions are independent. This assumption means that the cost of travel method (online hailing or traditional) will not affect whether passengers travel or not or the choice of destination. The purpose of this assumption is to remove the new travel demand caused by the emergence of online car-hailing, so as to simplify our estimation. Secondly, passengers will choose and optimize their travel method rationally. Specifically, we assume that people will optimize their travel decisions in the following three aspects: the overall travel time, the uncertainty of travel time, and the economic cost of travel. In other words, people want to choose the travel method with less time-consuming, higher certainty (accurate estimation of arrival time) and lower cost. This assumption excludes complicated factors in the choice of travel method, such as the comfort level of vehicles, whether it is necessary to change buses or walk halfway, the complexity of security check, etc. In the final robustness test, we will discuss other factors outside the model that may have an important impact on travel decision-making.

Following the literature, it is assumed that passengers follow this relative risk avoidance utility function (CRRA),

$$
U(w)=E(w)-\frac{\alpha}{2} * \operatorname{var}(w) .
$$

In this formula, $w$ represents the wage rate of passengers and $\alpha$ represents the coefficient of risk aversion. $\alpha$ is generally greater than zero (generally $2-5$ ), indicating that people are risk-averse, and that all people have the same degree of risk aversion. The total time available for work and transportation in a day is $\mathrm{T}$, the time spent in the $i$ type of transportation on day $\mathrm{d}$ is $t_{d}^{i}$, and the cost incurred is $f_{d}^{i}$.

Under the above assumptions, we get the following utility function of online car-hailing passengers:

$$
\operatorname{Max}_{i=1,2 . .}\left[w^{\star}\left(T-E\left(t_{d}^{i}\right)\right)-\frac{\alpha}{2}{ }^{\star} \operatorname{var}\left(t_{d}^{i}\right) \star w^{2}-f_{d}^{i}\right] .
$$

In Eq. (1), on day $d$, each passenger can maximize his or her utility by choosing a certain means of transportation $i$. As mentioned in our previous hypothesis, people have a variety of transportation options, such as bus, subway, car-hailing, etc. In order to maximize the utility, people balance the total travel time, travel time uncertainty and travel economic cost. If people choose the $i$ type of transportation on day $d$, a traffic time $t_{d}^{i}$ will be realized. It is worth noting that the running time $t_{d}^{i}$ of various means of transportation is time-varying. For example, the travel time of online car-hailing will decrease with the expansion of the scale of the online car-hailing platform and the accumulation of network advantages.

The expected value of traffic time is $E\left(t_{d}^{i}\right)$, and the variance is $\operatorname{var}\left(t_{d}^{i}\right)$. We assume that people's working time and travel time are completely replaceable, that is, people can put the saved travel time into work to generate value. If people choose the $i$ mode of transportation, then their working time is $\left(T-E\left(t_{d}^{i}\right)\right)$, so the amount of wages they get during this period is $w^{\star}\left(T-E\left(t_{d}^{i}\right)\right)$. 
At the same time, people's risk aversion means that choosing the $i$ type of transportation on day $d$ will produce the uncertainty of $\operatorname{var}\left(t_{d}^{i}\right)$, which will bring about the negative effect of $\frac{\alpha}{2}{ }^{\star} \operatorname{var}\left(t_{d}^{i}\right) \star w^{2}$. This term comes from the product of the sum of squares of wage $w^{\star}\left(T-E\left(t_{d}^{i}\right)\right)$ and $\frac{\alpha}{2}$. It is worth noting that the negative effects of this uncertainty will increase with the rise in wages. In other words, for those with higher wage per unit time, uncertainty becomes more unacceptable and has a greater negative impact on utility. The setting of our model itself may underestimate the benefits of time certainty, because this study ignores certain extreme scenarios where being on time is crucially important, e.g. catching a plane or a train, or being punctual at work.

The cost $f_{d}^{i}$ of each vehicle $i$ is also time-varying. The passengers therefore make the transportation choice to optimize the deterministic income $\left(w^{\star}\left(T-t_{d}^{i}\right)-\frac{\alpha}{2}{ }^{\star} \operatorname{var}\left(t_{d}^{i}\right) \star w^{2}\right)$ of working hours and at the same time minimize the transportation cost $f_{d}^{i}$.

When consumers turn from the $i$ vehicle to the $j$ vehicle, we assume the passenger is indifferent between the two:

$$
w^{\star}\left(T-t_{d d}^{i}\right)-\frac{\alpha}{2}{ }^{\star} \operatorname{var}\left(t_{d d}^{i}\right) \star w^{2}-f_{d d}^{i}=w^{\star}\left(T-t_{d d}^{j}\right)-\frac{\alpha}{2} \star \operatorname{var}\left(t_{d d}^{j}\right) \star w^{2}-f_{d d}^{j} .
$$

Let the $i$ be the traditional transportation, and the $j$ be the online car-hailing. This Eq. (2) means that on a certain day $d d$, passengers find that the consumer surplus they get by using the traditional means of transportation $i$ is equal to the consumer surplus of the new means of transportation $j$, the online car-hailing. Although the price of online car-hailing is higher $\left(f_{d d}^{j}>f_{d d}^{i}\right)$, it takes less time and has higher certainty. The time saved by the online hailing, given the current wage rate of passengers, just makes up for the high price, resulting in equal consumer surplus created by the two methods.

We assume that people make only one decision about whether to enter the online carhailing market. In other words, once consumers start to use online car-hailing, they will continue to use it. The purpose of this assumption is to avoid the complexity of the algorithm when people frequently - but not always - access the online car-hailing market. This assumption may be plausible for the following reasons. On one hand, the prices of various of transportation (including online car-hailing) are regulated in China, so they will remain unchanged for a long time. On the other hand, the travel time and uncertainty of public transport remain unchanged, while the travel time and uncertainty of online car-hailing will decrease with increased market scale and stronger agglomeration effect due to larger network cover by the online hailing platform. Moreover, as China's economy has been developing for a long time, the disposable income per capita has generally improved, in favor of online car-hailing.

For people with different wage rates, the time when they enter the online car-hailing market should be determined by the following equation:

$$
w_{d d}=\arg \left[w^{\star}\left(T-t_{d d}^{i}\right)-\frac{\alpha}{2} \star \operatorname{var}\left(t_{d d}^{i}\right) \star w^{2}-f_{d d}^{i}=w^{\star}\left(T-t_{d d}^{j}\right)-\frac{\alpha}{2} \star \operatorname{var}\left(t_{d d}^{j}\right) \star w^{2}-f_{d d}^{j}\right] \text {. (3) }
$$

The above equation shows that for those who enter the online car-hailing market on $d d$ day, their wage rate $w_{d d}$ is just equal to the level at which the consumer welfare created by 
online car-hailing can be equal to that created by traditional transportation. Those with a higher wage rate will enter the online car-hailing market before $d d$ day, because the time saved by online car-hailing is higher; those with a lower wage rate will enter the market later, or will not have entered the online car-hailing market so far, because the time saved by online car-hailing and the value created are not enough to make up for the higher price of online car-hailing. This allows us to extrapolate the implied wage rate for passengers from an observable behavior: the date when people enter the online car-hailing market.

Our above estimation method may underestimate consumer surplus. The main reasons are as follows: first, we may underestimate the time value of some early passengers entering the online car-hailing market, and then underestimate the consumer surplus. For those early passengers, we cannot deduce their implied wage rate because their wage rate is far higher than the cost of online hailing so that they enter immediately once the business is launched. Second, there is a certain switching cost when people choose their means of transportation. Various reasons, including the aversion to uncertainty, make people choose to enter the market only when they realize that the welfare effect of online car-hailing is significantly higher than that of traditional travel modes. Our model cannot fully capture this "welfare gap" and underestimates the consumer surplus generated by online car-hailing.

Once we get the implicit time value of consumers, we can get the social welfare that people obtain with the increase of scale of online car-hailing. Specifically, assuming that the car fare remains unchanged, the wage rate remains unchanged, and people do not quit after entering the online car-hailing market, then the calculation formula of consumers' social welfare can be expressed as follows:

$$
w_{d d} *\left(t_{d d}^{j}-t_{d f}^{j}\right)-\frac{\alpha}{2} *\left[\operatorname{var}\left(t_{d d}^{j}\right)-\operatorname{var}\left(t_{d f}^{j}\right)\right] \star\left(w_{d d}\right)^{2} .
$$

In Eq. (4), $t_{d f}^{j}$ represents the time spent on a certain day in the future $d f$, i.e. the trip of type $j$. The total social welfare consists of two parts: the decrease of travel time $\left(t_{d d}^{j}-t_{d f}^{j}\right)$ and the decrease of uncertainty $\operatorname{var}\left(t_{d d}^{j}\right)-\operatorname{var}\left(t_{d f}^{j}\right)$.

\subsection{Estimation method}

In order to estimate the social welfare of consumers, we first need to calculate the wage rate level from the time when consumers enter the online car-hailing market. For this reason, we estimate $\mathrm{c}$ for each city as follows:

$$
\left\{\begin{array}{l}
E\left(t_{d}^{j}\right)=\alpha_{1}^{c}+\text { Driver }_{d} \beta_{1}^{c}+X_{d} \gamma_{1}^{c}+\varepsilon_{d} \\
\operatorname{Var}\left(t_{d}^{j}\right)=\alpha_{2}^{c}+\text { Driver }_{d} \beta_{2}^{c}+X_{d} \gamma_{2}^{c}+\varepsilon_{d} \\
E\left(\text { Passager }_{d}^{j}\right)=\alpha_{3}^{c}+\text { Driver }_{d} \beta_{3}^{c}+X_{d} \gamma_{3}^{c}+\varepsilon_{d}
\end{array} .\right.
$$

In the above Equation, Driver ${ }_{d}$ is the total number of online car-hailing drivers on that day, which is a state variable and represents the scale of the online car-hailing platform. The three explainatary variables in Eq. (5) are the waiting time $E\left(t_{d}^{j}\right)$, the uncertainty $\operatorname{Var}\left(t_{d}^{j}\right)$ and the total number $E\left(\right.$ Passager $\left._{d}^{j}\right)$ of online car-hailing passengers on day $d$. We also add a series of control variables to control other factors that may affect the results. Specifically, the 
control variable $x_{d}$ mainly includes the annual fixed effect and the traffic item between the annual fixed effect and the number of drivers. The year fixed effect mainly controls for the change of traffic environment, and the interactive term of year fixed effect and the number of drivers means that we allow the influence of the number of drivers on the waiting time of passengers to vary from year to year.

Using the simple OLS regression between the daily passenger number and waiting time of the national online car-hailing platform from 2016 to 2018 , we can see that with the continuous expansion of the online car-hailing market, the number of passengers is increasing, and the average waiting time and waiting time uncertainty of each passenger are significantly reduced, as shown in Figure 1 and Figure 2.

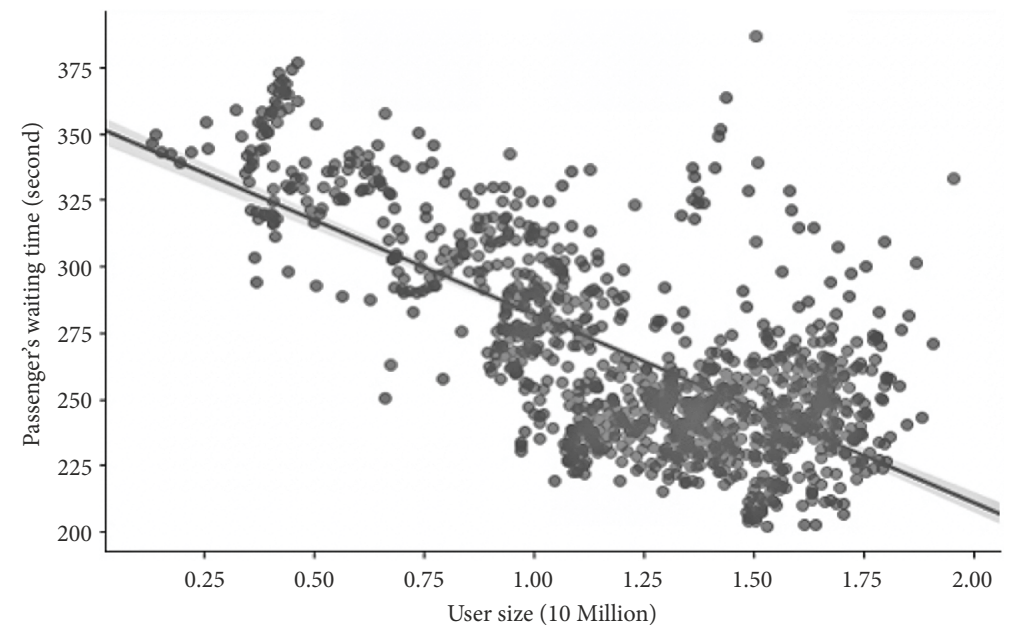

Figure 1. Relationship between user size and waiting time of passengers

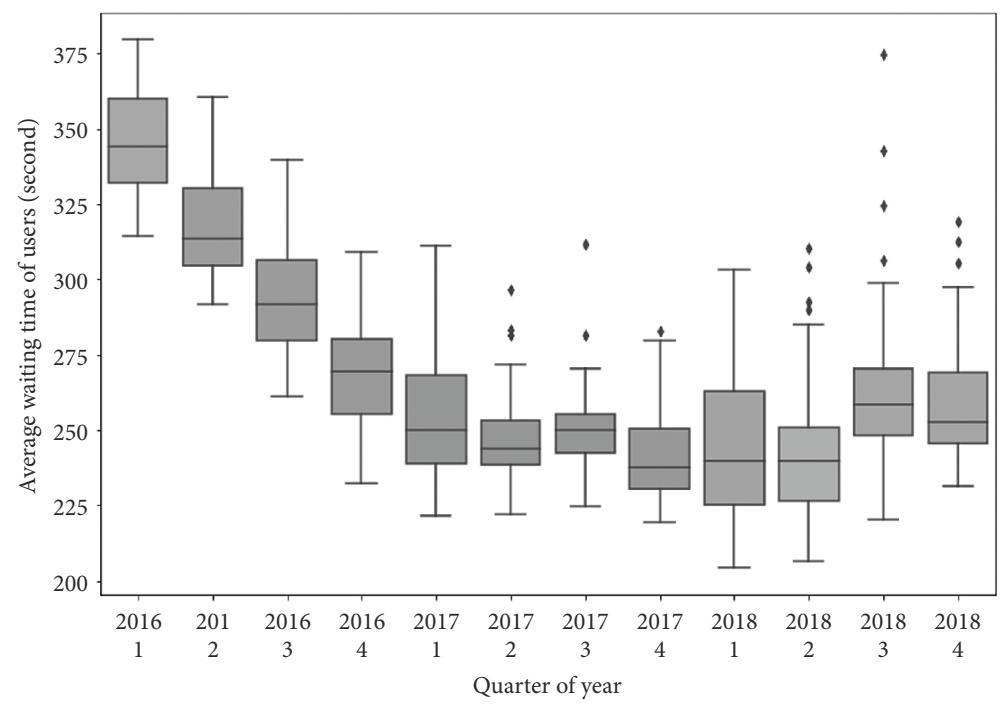

Figure 2. Variation of the average waiting time difference of passengers 
For a city $c$, we have the following linear relationship: the change of waiting time and waiting uncertainty can be expressed as a linear function of the scale of theonline car-hailing platform (number of users):

$$
\left\{\begin{array}{l}
\Delta E\left(t_{d}^{j}\right)=\Delta\left(\frac{\beta_{1}^{c}}{\beta_{3}^{c}}\right) E\left(\text { Passager }_{d}^{j}\right) \\
\Delta \operatorname{Var}\left(t_{d}^{j}\right)=\Delta\left(\frac{\beta_{2}^{c}}{\beta_{3}^{c}}\right) E\left(\text { Passager }_{d}^{j}\right)
\end{array} .\right.
$$

In order to simplify the calculation, we divide the online car-hailing users of the whole city into $\mathrm{n}$ groups, and the number of users in each group is equal to $1 / \mathrm{n}$ of the total number of users. We assume that each group of online car-hailing users is homogeneous, and they each have the same wage rate. Correspondingly, they enter the online car-hailing market at the same time. Obviously, as the number of groups increases, our calculation of overall social welfare will be more accurate, and we will discuss this effect in the robustness analysis.

Equation (6) gives us the average waiting time and the uncertainty of waiting time faced by the newly-entered group at the time when they enter the online car-hailing market. We use Eq. (3) to calculate the wage rate of this network car-hailing user $w_{d d}^{c, g}$. It represents the wage rate calculated for the passengers in the gth group of city $c$ at the time of entering the market. We then replace it with (4) and get the consumer surplus of the city's passengers of $g$ th group on $d f$ day. Finally, we add all consumers (different groups, different cities, and different times) to get the total consumer surplus of the online car-hailing platform $S_{c, g, d}$

$$
S_{c, g, d}=\sum_{c}\left[\left(\sum_{d d}^{d f} S_{d}\right) \star N_{g}\right] .
$$

Of which, $S_{d}=w_{d d}^{c, g} *\left(t_{d d}^{j}-t_{d f}^{j}\right)-\frac{\alpha}{2} *\left[\operatorname{var}\left(t_{d d}^{j}\right)-\operatorname{var}\left(t_{d f}^{j}\right)\right] *\left(w_{d d}^{c, g}\right)^{2}$.

Equation (7) denotes the additional benefits brought by consumers on the day of $t_{d f}^{j}$ due to the choice of the $j$-type transport (i.e. online car-hailing). We do similar calculations from day $t_{d d}^{j}$ (starting from consumers' entry into the market) to day $t_{d f}^{j}$, and add them to obtain the benefits of online ride-hailing during the whole period. Again, we assume that the consumers in group $g$ are homogeneous, so we multiply the number $N_{g}$ of group g to obtain the benefits of online ride-hailing for consumers in group $g$. Then we add all groups, to get the city c online car-hailing consumer surplus. Finally, the total consumer surplus created by the online car-hailing platform is obtained by summing the welfare of all cities.

It is worth noticing that we use the sub-group of the total population only when estimating their wage level, one variable that is related to their reserved price. When calculating the total welfare enhancement of the platform, we sum up the welfare of each individual order from the platform.

As for the estimation of producer surplus, because it is similar to the method and procedure of consumer surplus estimation, we do not repeat it in the main text. Drivers and consumers are symmetric in terms of welfare enhancement in many ways. Firstly, their welfare or surplus are both the difference between their actual transaction price and their reserve price. 
Moreover, they both benefit from the total travel time reduction brought up by the increased economy of scale of the ride-sharing platform. It's just that ride-hailing drivers don't benefit as much from reducing trip uncertainty as passengers do.

\subsection{Our method compared with other papers}

There are mainly two papers estimating the welfare effect of the ride-sharing platforms. The first one is the work of Cohen et al. (2016). It estimates the demand elasticity of the passengers using one unique feature of the Uber platform: its price surge during traffic time. For instance, during the traffic time, the Uber platform may suggest a $20 \%$ rise in price and the passenger may agree with it or not. If the passenger accepts the price surge, it means that the reserved prices of the passenger must be higher than the $20 \%$ over the market price. Therefore, with the information of the passenger acceptance of the rise in price or not, the coauthors estimate the reserved price and the demand elasticity. One serious concern of this estimate is that the places with a price surge may be systematically different from the places without a price surge: it could be the case that the supply is in shortage. Therefore, this method risk overestimating the surplus of the passengers. Although the coauthors use the Propensity Score Matching) (PSM) methods for correct the bias, there is no guarantee that the treatment and control group are matched across all features that may affect the passengers' demand. That is why the results of Cohen et al. (2016) are widely criticized for their upward bias.

The second paper is that of Lam and Liu (2017). They face a similar endogeneity issue. They make use of the instrumental variable. Specifically, they use the prices of all taxies that arrive in a focal place as the instrumental variable of the local supply of rides. The reason is that if the price is low, then people are more likely to go to the focal point, therefore increasing the supply and depressing the price of the next journey. However, the exclusive restriction of the instrumental variable may be violated, as the price of the last trip is to a great extent related to its destination, which is related to the local demand.

One common feature of the above two papers is that they both use a very short sample period and a limited number of cities. Lam and Liu (2017) used only one-month data for New York City, while Cohen et al. used six-month data for four cities. Our sample covers more than 300 cities for almost 3 years. The long sample period and the comprehensive coverage make it possible for us to estimate the enhancement of social welfare that comes from the increase in the economic efficiency of the ride-sharing platform over time.

Another important issue is that both aforementioned papers estimate the social welfare, rather than the additional social welfare created by the ride-sharing platform, as estimated by our model. For instance, if one passenger switch from using a taxi to online ride-sharing, any difference between the actual Uber price and his reserved price would be captured by the social welfare, but not the additional social welfare. That is because using the taxi could generate the same consumer surplus but only be crowded out by the ride-sharing. It is plausible that estimating this additional social welfare has more important policy implications. 


\section{Empirical analysis and results}

The data for online ride-hailing transactions used in this paper come from a large national online ride-hailing travel platform. The platform was launched in 2012. In the early stage, it mainly cooperated with taxis. In 2015, it launched the online hailing business providing private car drivers. At present, the platform accounts for more than $80 \%$ of China's online car-ride market. We use the transaction-level data of all express cars on the ride-hailing platform on the Internet from 2016 to 2018. The express cars are the low-end product on the platform. The advantage of using express car data is that the orders are huge, with an average of more than 20 million orders per day. The huge order size enables us to get more accurate social welfare estimates. The express order data in this paper covers 359 major cities in China, including all major cities and large counties. We mainly extract and use the daily express order quantity, passenger traffic time and variance data of each city. In order to compare with traditional means of transport, we also collected and used data on average speeds and corresponding costs of other public transport in cities such as subways and buses. Table 1 shows the sample descriptive statistics of one representative city generated by random sampling.

Table 1. Description statistics of annual online car-hailing in a city

\begin{tabular}{|l|c|c|c|}
\hline \multicolumn{1}{|c|}{ Particular year } & 2016 & 2017 & 2018 \\
\hline Total orders (10,000) & 6585 & 8375 & 9493 \\
\hline Total drivers (10,000) & 19 & 16 & 14 \\
\hline The proportion of full-time drivers & $29 \%$ & $36 \%$ & $41 \%$ \\
\hline The proportion of part-time drivers & $71 \%$ & $64 \%$ & $59 \%$ \\
\hline Number of passengers (10,000) & 338 & 500 & 614 \\
\hline Average waiting time (seconds) & 327 & 288 & 303 \\
\hline Average cost (Yuan) & 12 & 17 & 19 \\
\hline
\end{tabular}

Table 1 shows that the online car-hailing market has shown an expansion trend in the last three years (2016-2018). The total amount of orders, the total number of passengers and the average cost of passengers support this conclusion. The total number of express orders in 2018 increased by $44 \%$ compared with 2016, and the number of passengers increased by $82 \%$. The results in Table 1 also show that online car-hailing regulation has a significant impact on the development of the online car-hailing market. After strict regulation in 2018, the total number of online car-hailing drivers fell by more than $20 \%$ from 2016, with full-time drivers falling from $71 \%$ in 2016 to $59 \%$ in 2018 . We also note that the average waiting time for passengers in 2018 was significantly higher than in the previous year. These results show that the strong regulation of online ride-hailing implemented by local governments since 2018 has a significant impact on the online ride-hailing market and the social welfare created.

\subsection{Baseline estimation results}

Pursuant to the estimation method described in Section II, we obtain the estimation results of the social welfare of the online car-hailing platform as shown in Table 2. The results of 
Table 2 show that the total social welfare generated by the online car-hailing platform from 2016 to 2018 was 130.5 billion Yuan, of which the social welfare attributed to consumers was 88.6 billion Yuan and the welfare attributed to producers was 41.8 billion Yuan. At the same time, the network effect of online car-hailing saved passengers more than 1.7 billion hours of travel time, equivalent to 850,000 person-years. Both the total social welfare generated by the online car-hailing platform and the time saved for passengers are very high figures, indicating that the entire online car-hailing industry has brought huge positive externalities to the society. We also calculate the consumer surplus and producer surplus from a single transaction, which are 5.4 Yuan and 2.5 Yuan respectively. Considering the average price of a single transaction is 15 Yuan, each dollar transaction can produce 0.3 Yuan consumer surplus. The number is significantly less than that of Cohen et al. (2016), where each dollar transaction can produce 1.6 dollars consumer surplus. The reason for this difference is probably due to the average disposable income level of residents in China. Moreover, our estimation method is more conservative than that of Cohen et al. (2016). A lower disposable income means China's online ride-hailing price elasticity is low. This 5.4-Yuan consumer surplus means that if the online car-hailing driver increases a 15 Yuan order to 20.4 Yuan, then passengers are likely to choose other means of transport because it is too expensive, which is intuitive. Cohen et al. (2016) estimate that passengers are willing to pay an average of $\$ 52$ for a $\$ 20$ order, an estimate which seems too high.

Table 2. Baseline estimation results (100 million Yuan)

\begin{tabular}{|l|c|c|c|c|}
\hline \multicolumn{1}{|c|}{ Classification by group } & subtotal & 2016 & 2017 & 2018 \\
\hline Grand total & 1304.9 & 286 & 541.7 & 477.3 \\
\hline Producer & 418.6 & 91.6 & 174.3 & 152.7 \\
\hline Consumer & 886.4 & 194.4 & 367.4 & 324.6 \\
\hline First-order effect & 768.5 & 174.7 & 308.5 & 285.3 \\
\hline Second-order effect & 117.9 & 19.7 & 58.9 & 39.3 \\
\hline
\end{tabular}

From the results in Table 2, it can be seen that the creation of social welfare on online car-hailing platforms is asymmetrical between producers and consumers: the corresponding welfare of consumers is almost twice that of producers. For various reasons, Chinese passengers' travel needs are not being meet by traditional travel method, such as subways, buses and taxis, so the emergence of online car-hailing will bring them huge social benefits. In contrast, the producer surplus is not as large as the consumer suplus, probably due to the fact that the drivers usually have other job opportunities. At the same time, the data also show that online ride-hailing plays a greater role in reducing passenger travel time than reducing travel uncertainty. One possible explanation is that in the model we do not consider the extreme scenarios where people have to be punctual, e.g. catching a flight or a train.

\subsection{Inter-city heterogeneity results}

The conclusion in Table 2 is based on nationwide estimation. There are great cross-city differences in the level of economic development, residents' consumption level, wage rate level, 
travel convenience and the operation of online car-hailing platform, and that may bring great differences in the social welfare of online car-hailing among cities. Therefore, we estimate the social welfare creation of online ride-hailing in first-, second- and third-tiered cities. First-tier cities include Beijing, Shanghai, Guangzhou and Shenzhen. Second-tier cities include Chengdu, Hangzhou, Chongqing, Wuhan, Suzhou, Xi'an, Tianjin, Nanjing, Zhengzhou, Changsha, Shenyang, Qingdao, Ningbo, Dongguan, Wuxi and others. Third-tier include all other cities ${ }^{1}$. The estimated results are shown in Table 3.

Table 3. Social welfare creation in large, medium and small cities (100 million Yuan)

\begin{tabular}{|l|c|c|c|c|}
\hline \multicolumn{1}{|c|}{ Classification by group } & subtotal & 2016 & 2017 & 2018 \\
\hline First-tier cities & 353.6 & 52.02 & 191.87 & 109.71 \\
\hline Producer & 109.86 & 15.55 & 53.42 & 30.89 \\
\hline Consumer & 243.74 & 36.47 & 138.46 & 78.81 \\
\hline Second-tier cities & 655.5 & 162.85 & 237.92 & 254.73 \\
\hline Producer & 153.75 & 36.71 & 61.95 & 55.10 \\
\hline Consumer & 501.75 & 126.15 & 175.97 & 199.63 \\
\hline Third-tier cities & 285.8 & 71.09 & 164.82 & 49.89 \\
\hline Producer & 95.00 & 20.97 & 59.64 & 14.38 \\
\hline Consumer & 190.80 & 50.12 & 105.18 & 35.51 \\
\hline
\end{tabular}

The results in Table 3 show that there are great differences in the total amount of social welfare creation among cities. The total social welfare of the online car-hailing platform in first tier cities in 2016-2018 was 8.84 billion Yuan, while the average value in a second-tier city was 4.37 billion Yuan, and the total social welfare in a third-tier city was only 105 million Yuan. This cross-city heterogeneity is first related to our classification method. Large and medium-sized cities are the largest, most populous and most economically developed cities. With larger population and higher per capita income, online car orders will also be larger accordingly. Another possible reason for heterogeneity is that many big cities, such as Beijing and Shanghai, have strict restrictions on the purchase of private cars, a measure to control the total amount of traffic. Riding online is thus a very important travel choice. For many thirdtier cities, purchasing power is not high, city size is not large, and roads are not crowded. Here, online ride-hailing travel is often replaced by private cars, bicycles or electric bicycles.

\subsection{Regulatory estimates of social welfare losses}

With the outbreak of several online car-hailing security incidents in 2018, cities have introduced more stringent regulatory measures for online car-hailing businesses and platforms. These regulatory measures have brought about a rapid decline in social welfare created by online car-hailing. Those regulations impose restrictions on the drivers, the vehicles and the platforms that are engaged in the car-hailing businesses. Most cities require that only drivers with local residency can drive the online hailing cars. This greatly decreased the total

\footnotetext{
${ }^{1}$ Our criteria for dividing large, medium and small cities refer to 2019 Urban Business Charm List of First finance.
} 
number of qualified drivers, as before the regulation, most drivers were low-income migrant workers. Only those drivers who pass the qualification test are now allowed to operate. But local governments usually deliberately reduce the tests slots to delay the qualification of drivers. On the regulations of the vehicles, most cities set the floors of the car displacement, excluding the smaller cars, the largest share of the operating vehicles, from the market. Local administrations also forbid cross-region operations, declaring that all platforms that are not registered and paying taxes locally are illegal.

We firstly make a relatively conservative estimate of the welfare loss caused by the regulation of online car-hailing. We assume that in any city, in the absence of these regulatory measures, the growth rate of online car-hailing is zero, and the negative value can only be due to strict regulation. Considering the massive demand for online car-hailing, the advance assumption should be in line with the actual situation. We compare the difference between the number of online car-hailings in each city at each time point after the introduction of strict regulation and the maximum value before the city's strict regulation. If this difference is a negative number, we believe that this value is due to the loss of social welfare caused by regulation. Our estimation method is very conservative because we assume that the growth rate without supervision is 0 . In reality, the growth rate of online car-hailing orders without supervision may be a relatively large value. For example, in the loosely regulated period 2016-2017, online car-hailing social welfare grew by nearly $100 \%$. Our estimation results are shown in Table 4.

Table 4. Social welfare losses caused by online car-hailing supervision (100 million Yuan)

\begin{tabular}{|l|c|l|c|}
\hline \multicolumn{2}{|c|}{ Total } & \multicolumn{2}{c|}{189.1} \\
\hline Full-time driver & 125.2 & Part-time driver & 63.9 \\
\hline Producer & 34.8 & Producer & 20.9 \\
\hline Consumer & 90.5 & Consumer & 43.0 \\
\hline
\end{tabular}

The evidence from the time dimension also shows the impact of regulation on online car-hailing. In 2017, online car-hailing social welfare is the highest, while in 2018 it has declined. This decline is not a signal of market saturation. On the contrary, there are very many travel needs that have not been met. The strong regulatory measures in 2018 stopped the trend of rapid development of online car-hailing, and the total social welfare created by online car-hailing also significantly decreased. In 2018, the social welfare loss of online car-hailing reached 20.5 billion Yuan, including 14.5 billion Yuan for consumer welfare and 6 billion Yuan for producer welfare. This figure is close to the sum of social benefits generated by the platform in 2016. In addition, we found that part-time drivers can improve the response efficiency of online car-hailing to a greater extent, which is likely to be related to the more flexible operation time of part-time drivers.

This conclusion has rich policy implications. In the process of introducing online carhailing regulatory policy, regulators need to weigh its impact on social welfare creation while considering other social problems such as safety and traffic problems. 


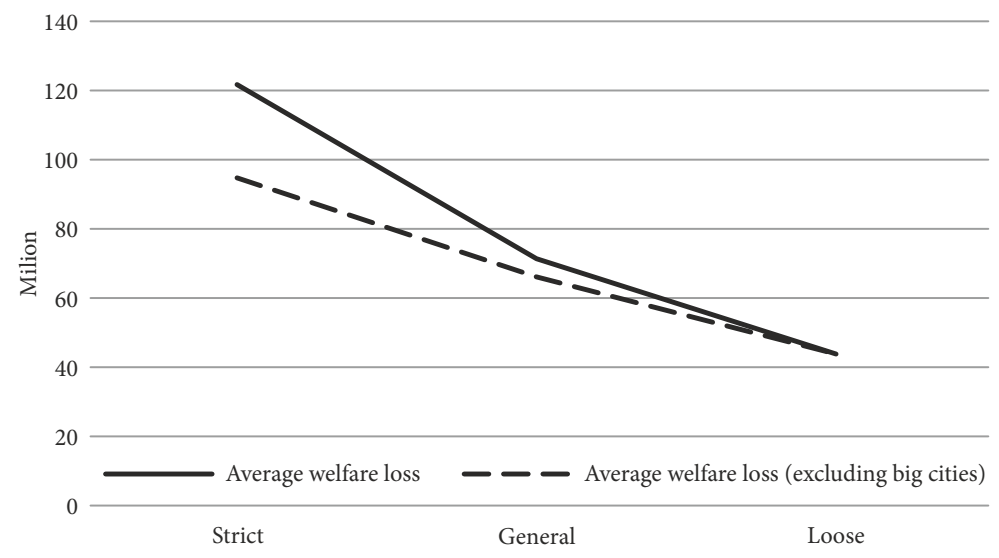

Figure 3. Regulatory intensity and welfare losses

In order to further understand the impact of regulation on the social welfare of online car-hailing, we divide the government's online car-hailing regulation into three categories according to the severity of its regulation: severe, general and loose, and then calculate the total social welfare in these three cities ${ }^{2}$. We find that there is a significant positive relationship between regulatory intensity and welfare loss: cities with higher access threshold and stricter regulation have more welfare losses, as shown in Figure 3. A natural question is whether those cities with strict supervision have some common characteristics, such as size. In big cities, the number of benefits generated by online ride-hailing is large, so it is not surprising that the losses after regulation are greater. In response to this concern, we also give the results after omitting big cities. After removing big cities from our analysis, regulatory intensity and welfare losses remain negative. The regulatory implication of this is that the principle of prudence should be adhered to in the supervision of online car-hailing. Blindly strict supervision will have a significant negative impact on social welfare.

\section{Robustness test}

In the previous section, we made a series of assumptions when estimating the social welfare of online car-hailing, and changing these assumptions may affect our final estimation results. In order to evaluate the impact of the change of assumptions on the estimation results, we conduct the following robustness tests.

\subsection{Robustness test of parameters}

We first investigate whether the estimation results are affected by the selection of parameter $\alpha$ in the model. $\alpha$ represents the degree of people's risk aversion in the utility function, and the

\footnotetext{
${ }^{2}$ The decision as to whether online car-hailing policy is loose or not follows the corresponding distinction method of China's online car-hailing policy implementation and research report (2018) released by the Chinese Academy of Information and Communications. This report divides the policies of each city according to whether household registration is required, whether there are requirements regarding vehicle axle distance, displacement, or age, and other factors. There are 26 cities with loose policies, 116 cities with policies between loose and severe, and 66 cities with severe policies.
} 
higher the value is, the greater the degree of people's risk aversion is. In our online car-hailing welfare model, the higher $\alpha$ value means that people are more sensitive to the uncertainty of online car-hailing commuting time. Thus, when travel uncertainty is reduced, people's benefits increase accordingly. At the same time, changes in $\alpha$ may indirectly affect our estimates of social welfare. When people pay more attention to travel uncertainty, our estimation of people's wage rates may be affected, thus affecting the estimation of social welfare created by reduced travel time.

Generally speaking, the value range of $\alpha$ is $2-5$, and the risk aversion close to 10 will be considered too high. In the baseline estimation, we set the value of $\alpha$ as 2 , and for the robustness test we set $\alpha=5$. The use of a large degree of risk aversion may be justified by the nature of the demand for online ride-hailing in China. A considerable share of online ride-hailing in China is commuting to work. One of the characteristics of commuting demand is that the demand for arriving at the destination on time is quite important, as most companies in China do not implement flexible working systems. Therefore, the high degree of aversion to online ride-hailing travel uncertainty may be a common feature of online ride-hailing passengers' decision-making in China.

Table 5. Social welfare creation under high-risk aversion $(\alpha=5$; Billion Yuan)

\begin{tabular}{|l|c|c|c|c|}
\hline \multicolumn{1}{|c|}{ Classification by group } & subtotal & 2016 & 2017 & 2018 \\
\hline Total & 108.9 & 23.2 & 46.3 & 39.4 \\
\hline Producer & 36.7 & 7.9 & 14.2 & 14.5 \\
\hline Consumer & 72.2 & 15.3 & 32.0 & 24.8 \\
\hline First-order effect & 59.9 & 13.3 & 26.0 & 20.6 \\
\hline Second-order effect & 12.3 & 2.0 & 6.0 & 4.2 \\
\hline
\end{tabular}

Table 5 shows the estimated results of social welfare creation of online car-hailing after increasing risk aversion. When we adjust the risk aversion value to 5 , all the remaining results of consumers have changed, which is consistent with our expectations. With the increase of risk aversion, the social welfare obtained by reducing travel uncertainty has increased from 11.79 billion Yuan to 12.3 billion Yuan, a significant increase. At the same time, the social welfare obtained by people from reducing travel time has been significantly reduced from 76.9 billion Yuan to 59.9 billion Yuan.

We also note that most of the results estimated above have not changed significantly. The total amount of social welfare has changed from 130.5 billion Yuan to 108.98 billion Yuan, and the total amount of social welfare creation remains a considerable amount. At the same time, the each share of social welfare created in 2016-2018 has not changed significantly. This shows that our choice of the risk aversion coefficient can only partially affect our estimates and does not affect the overall pattern in our baseline tests.

\subsection{Robustness test of user segmentation}

Our second robustness test is to change the segmentation method for each city's online car users. As mentioned earlier, we divide each city's online car-hailing users by $\mathrm{n}$, and then 
estimate the wage rate for each group. It is obvious that improving the classification number of urban online car users $n$ can improve our estimation accuracy. An ideal case is that if $n$ is equal to the total number of online car-hailing users in the city, then we can accurately calculate the wage rate of each online car-hailing user. But at the same time, more user segmentation also brings challenges to our computing ability. Therefore, an important problem is to find a suitable number for user classification. Here, we use a user segmentation method with 20 , instead of 10 groups. If the results obtained by this method are similar to those obtained by using 10 groups, then we are more confident that the user segmentation method in the baseline estimation is feasible. We assume that the accuracy gain of the results is gradually decreasing with the improvement of the total number of sample divisions. That is to say, the accuracy gain between 10 groups to 20 groups should be higher than that of 20 groups to 30 groups. Therefore, if we prove that the effect of 10-score to 20-score on the results is very small, the effect of higher precision division on the estimation results will not be very pronounced.

Table 6. Robustness test of user segmentation method ( $\mathrm{n}=20$; billion Yuan)

\begin{tabular}{|l|c|c|c|c|}
\hline \multicolumn{1}{|c|}{ Classification by group } & subtotal & 2016 & 2017 & 2018 \\
\hline Grand total & 116.5 & 25.3 & 52.6 & 38.6 \\
\hline Producer & 35.8 & 8.4 & 15.7 & 11.7 \\
\hline Consumer & 80.7 & 16.9 & 36.9 & 26.9 \\
\hline First-order effect & 69.4 & 14.7 & 31.0 & 24.2 \\
\hline Second-order effect & 11.3 & 2.2 & 5.9 & 2.7 \\
\hline
\end{tabular}

For each city, we use the results of 20-group segmentation, as Table 6 shows. We find that more precise user classification does not have a substantial impact on our results. Although the estimates of some items are improved, the overall pattern obtained by the baseline regression, including time trends, and cross-sectional (producers, consumers) trends are consistent with the baseline estimates. This proves that the 10 - group user division method can accurately estimate the social welfare creation of online ride-hailing, and the more detailed division does not significantly affect our basic conclusion. (It also significantly increases our calculation requirements.)

The above results are probably related to the good linearity of our samples. The n-equalization of each city sample is actually based on the assumption that the sample has a linear nature. That is to say, the social welfare of those who are in the i representative point (for example, the time to enter the online car market is $50 \%$ of the whole city) and the $i+1$ representative point (for example, the time to enter the online car market is $60 \%$ of the whole city) can be obtained by linear interpolation of the social welfare of the corresponding people. The observed value (the time at entry into the online car market), and the value (social welfare) we inferred, will approximately maintain a linear relationship in a relatively small range. This linear property makes the research results insensitive to the number of interpolations. 


\subsection{Influence of nonlinear properties on estimation results}

In our estimation we assume that there is a linear relationship between variables. For example, in the process of looking for passenger scale, passenger travel time and travel uncertainty, we conduct a linear regression. We assume that those high-order nonlinear relations have little effect on our results. To verify the effect of this assumption on the estimation results, we modify the simultaneous equations used for estimation to the following form;

$$
\left\{\begin{array}{l}
E\left(t_{d}^{j}\right)=\alpha_{1}^{c}+\text { Driver }_{d} \beta_{1}^{c}+\left(\text { Driver }_{d}\right)^{2} \rho_{1}^{c}+X_{d} \gamma_{1}^{c}+\varepsilon_{d} \\
\operatorname{Var}\left(t_{d}^{j}\right)=\alpha_{2}^{c}+\text { Driver }_{d} \beta_{2}^{c}+\left(\text { Driver }_{d}\right)^{2} \rho_{2}^{c}+X_{d} \gamma_{2}^{c}+\varepsilon_{d} \\
E\left(\text { Passager }_{d}^{j}\right)=\alpha_{3}^{c}+\text { Driver }_{d} \beta_{3}^{c}+\left(\text { Driver }_{d}\right)^{2} \rho_{2}^{c}+X_{d} \gamma_{3}^{c}+\varepsilon_{d}
\end{array} .\right.
$$

In Equation (9), we add the quadratic term $\left(\text { Driver }_{d}\right)^{2}$ of the online car-hailing scale to capture various nonlinear relationships. If the nonlinear relationship is very significant, they will be reflected in the coefficient $\rho^{c}$. In this way, we can get the new relationship between $E\left(t_{d}^{j}\right), \operatorname{Var}\left(t_{d}^{j}\right)$, and $E\left(\right.$ Passager $\left._{d}^{j}\right)$. We then use Eq. (3) to solve the wage rate of that group of passengers, and then use formula (7) to sum up the social welfare of all passengers (drivers). The estimated results considering the nonlinear relationship of variables are shown in Table 7.

Table 7. Robustness test for nonlinear relations (billion Yuan)

\begin{tabular}{|l|c|c|c|c|}
\hline \multicolumn{1}{|c|}{ Classification by group } & subtotal & 2016 & 2017 & 2018 \\
\hline Grand total & 118.2 & 25.5 & 49.8 & 43.0 \\
\hline Producer & 37.8 & 8.3 & 16.0 & 13.4 \\
\hline Consumer & 80.4 & 17.1 & 33.8 & 29.5 \\
\hline First-order effect & 70.0 & 15.3 & 28.6 & 26.1 \\
\hline Second-order effect & 10.5 & 1.8 & 5.2 & 3.5 \\
\hline
\end{tabular}

It can be found that the estimation results after adding quadratic terms in the estimation model are roughly consistent with the baseline estimation results in Table 2. As mentioned above, the reason the quadratic term does not affect the results is likely to be related to the good linear nature of the data we used. In other words, the waiting time of passengers, the uncertainty of waiting, and the scale of passengers are all in a certain range to maintain a linear relationship with the scale of online ride-hailing.

\subsection{The learning effect}

The estimation of our baseline results is also based on an important assumption: Passengers do not enter the online car-hailing market mainly due to non-economic considerations. We assume that people fully understand the price of online car-hailing. The reason people don't choose online car-hailing is because of economic factors, that is, the time saved by online car-hailing is not equal to the economic cost. However, we may have overlooked another im- 
portant possibility. The reason passengers choose not to enter the online car-hailing market at at particular time point may be because they are not familiar with the online car-hailing process, or even that they do not know that it exists. People gradually begin using the platform not because it can improve their travel experience, or reduce the time cost or uncertainty, but because it's only gradually known to passengers. Some groups, such as the elderly, may be more affected by this factor than others. It should be admitted that in the early stage of the promotion of the online car-hailing platform, the increase of the scale of the platform, the entry of passengers, and the platform being known happen almost at the same time. If this kind of learning effect and people's economic considerations exist at the same time, we may overestimate the social welfare created by the online car-hailing platform if we include all people into the simple economic considerations.

We use the following methods to verify the impact of learning effect on baseline results. We examine the behavior of previously inactive online car-hailing users. Less active users refer to those who have registered online car-hailing accounts for a long time but have not used them frequently. The learning effect of these users should be relatively weak as they must have known about the online car-hailing platform as they registered long before. The reason they don't often use online car-hailing is more likely to be related to economic factors, that is, compared with traditional transportation, the cost of online hailing is too high.

In empirical analysis, we regard those passengers who have registered for online car booking but use make less than one order per month within the first year after registration as those who have already known about car-hailing. Then we use the data of this sub-sample to estimate their wage rate, and interpolate the final result to all online car bookings according to the corresponding wage rate level among customers, the estimated results shown in Table 8.

The results in Table 8 show that when we use the less active user samples to test the learning effect, although the final social welfare estimate slightly decreases, there is no significant difference from the baseline results in Table 2. These results show that the learning effect does exist, but it is not the main driving factor that causes people not to take the online car. People mainly make their own travel decisions based on economic reasons. In addition, we have found that the difference between the result considering the learning effect and our baseline results is most significant in 2016. This is because 2016 is the time when carhailing (the express service) went online on a large scale. With the expansion of the scale of online car-hailing, the increase of the number of passengers, the improvement of operating efficiency, and the gradual popularity of the service, all nearly simultaneously, the learning

Table 8. Impact of learning effect (billion Yuan)

\begin{tabular}{|l|c|c|c|c|}
\hline \multicolumn{1}{|c|}{ Classification by group } & subtotal & 2016 & 2017 & 2018 \\
\hline Grand total & 122.4 & 24.9 & 53.1 & 44.4 \\
\hline Producer & 38.0 & 7.3 & 17.1 & 13.6 \\
\hline Consumer & 84.4 & 17.6 & 36.0 & 30.8 \\
\hline First-order effect & 74.0 & 16.0 & 30.6 & 27.4 \\
\hline Second-order effect & 10.4 & 1.6 & 5.4 & 3.4 \\
\hline
\end{tabular}


effect is most obvious. In 2017, due to the strong publicity of online car-hailing, especially on Wechat, microblog and other social media, online car-hailing was widely known to its target audience, which relatively weakens the learning effect in online car-hailing. Therefore, the results in Table 9 are consistent with our intuition.

\subsection{The impact of bicycle-sharing on the estimated results}

Another assumption in our baseline estimation is that the reduction of online booking passenger travel times comes from the improvement of vehicle scheduling efficiency in the online booking platform. But in reality, there may be many reasons for this decline in travel time. Some factors, such as the improvement of road conditions in a single city, cannot affect the travel quality of online car-hailing users nationwide, but some global factors may affect all cities. The launch of bicycle sharing platforms is one such factor. Bicycle sharing solves the "last mile" problem in people's transportation by launching bicycle sharing service. Bicycle sharing has been promoted nationwide since 2016, and was launched in major cities in the spring of 2017. This period of time coincides with the period of vigorous promotion of online car-hailing. Therefore, does the introduction of bicycle sharing lead to the diversion of a large number of short-distance passengers, thus improving road driving conditions and reducing the travel efficiency of online car-hailing? If we do not control for this factor, we may overestimate the social welfare of online car-hailing.

In order to get more robust results, we need to exclude the impact of bicycle sharing on our results. Our strategy is to add the amount of bicycle sharing of a particular month into the baseline regression equation as the control variable. In other words, put the amount of bicycle sharing into $X_{d}$. If, as we have posited, bicycle sharing has a significant impact on road traffic, then this impact should be captured by $\gamma^{c}$, rather than reflected in the linear relationship between $E\left(t_{d}^{j}\right), \operatorname{Var}\left(t_{d}^{j}\right)$ and $E\left(\right.$ Passager $\left._{d}^{j}\right)$. According to this idea, we reevaluate Eq. (5) and report the corresponding estimation results in Table 9.

The results in Table 9 show that the estimated results after controlling for bicycle sharing effect in the regression equation are almost the same as the baseline results in Table 2. That is, the impact of bicycle sharing on private car traffic is not as significant as expected, and the coincidence range of bicycle sharing users and online car-hailing users may be small.

In summary, the baseline estimation results of this paper are robust to a series of robustness tests, including changing the risk aversion coefficient, changing the customer segmentation method, changing the linear property hypothesis, controlling for the introduction of

Table 9. Control for bicycle-sharing effect (billion Yuan)

\begin{tabular}{|l|c|c|c|c|}
\hline & subtotal & 2016 & 2017 & 2018 \\
\hline Grand total & 126.6 & 27.2 & 53.1 & 46.3 \\
\hline Producer & 40.6 & 9.1 & 16.7 & 14.8 \\
\hline Consumer & 85.9 & 18.1 & 36.4 & 31.5 \\
\hline First-order effect & 74.5 & 16.2 & 30.7 & 27.6 \\
\hline Second-order effect & 11.5 & 2.0 & 5.7 & 3.9 \\
\hline
\end{tabular}


bicycle sharing, or for people's learning effect, etc. We also carried out other further tests and analyses to make our results more robust and more in line with reality. For example, we considered the possibility that a large proportion of online car-hailing passengers (perhaps as great as $40 \%$ ) transferred from the taxi market, and considered the price and waiting time between online car-hailing and taxi-hailing, and estimated the corresponding social welfare, considering the possibility of additional travel demand caused by online car-hailing, and so on. In general, these changes have no significant impact on our baseline estimates ${ }^{3}$.

\subsection{Using taxis as baseline}

Taxis are largely similar to ride-hailing in terms of price. However, the waiting time and uncertainty of the taxis are quite high, decreasing the utility of the passengers. According to the Blue Book of Public Service, a joint research conducted by the Academy of Social Sciences, Huatu Zhengxin Institute of Public Administration, and Social Sciences Academic Press, more than $45 \%$ of respondents in Beijing suggest that they have to wait for 10-30 for a taxi and $8.5 \%$ have to spend more than 30 minutes. On the contrary, more than $95 \%$ of ride-sharing will get a car in 10 minutes. Therefore, even at the same price, online ridehailing can greatly improve social welfare in comparison with taxis. To verify this intuition, we repeat the baseline result using the taxi traveling and waiting time. The taxi waiting time comes from the aforementioned Blue Book of Public Service. The estimated result is quite similar to our baseline result using public transportation. It is probably not that surprising, as the market is in such an equilibrium that marginal travelers are indifferent between taxi and public transportation before the emerge of the ride-sharing services.

\subsection{Potential channels for negative impact on social welfare}

Admittedly, the online ride-hailing platform may decrease social welfare in several ways. The first one is that ride-hailing may aggregate the local traffic when passengers increasingly prefer ride-hailing over public transportation. Firstly, for most off-peak times, when the carrying capacity of the road network does not reach its limit, such a problem does not exist. Even for the peak time, the effect doesn't need to be negative, as ride-hailing may reduce the total number of people driving their own car. A small number of papers discussed this issue and the results are mixed (Gao et al., 2016; Yang et al., 2020).

Another possible way of welfare loss is the carbon emissions from ride-hailing traffic, which contribute to global warming. However this concern should be mitigated by the fact that many ride-hailing drivers now are driving electrical cars, which are significantly less costly, given the much lower electricity price compared with that of gasoline. Some local governments are also making efforts to promote electrical cars for ride-hailing traffic. For instance, the Shenzhen government requires that all ride-hailing cars must be electrical. With the continuous effort of adopting clean energy in China, the welfare loss caused by carbon emissions of ride-hailing traffic should be decreased over the next decade.

\footnotetext{
$\overline{{ }^{3} \text { Due to limited space, }}$ those results are not repeated here.
} 


\subsection{The effect of responsive rate of the platform}

Our assumption here is that all passenger needs that were sent to the platform are already met by ride-sharing vehicles. However that is not the case in reality. A great share of passengers canceled the trip before the driver arrives, either because they see a taxi coming by or they cancel the whole trip. The total number of canceled orders accounted for around $12 \%$ of the total orders. In practice, however, this share could be even under-estimated, as many people probably cancel the trip or use other types of transportation before they send their orders to the ride-sharing platform. We matched the orders that were closed to the canceled order in terms of starting point, destination and order time. We estimate the total welfare if all those canceled orders were fulfilled. We find that the total social welfare went up to 132 billion Yuan now.

\section{Research conclusions and policy recommendations}

This paper estimates the social welfare of online hailing in China, by using transaction-level data from a large national shared travel platform. We use the difference between the time of passengers entering the online car-hailing market and traditional transportation options to calculate the wage rate of passengers entering the market. Then we estimate the social welfare brought about by the decrease in waiting time and the decrease in waiting uncertainty caused by the increase of scale effect of online car-hailing after passengers enter the network. We show that the online hailing platform created 13.5 billion Yuan-worth of social welfare in the three years from 2016 to 2018, and that passengers saved more than 1.7 billion hours of travel time. The consumer surplus and producer surplus created by a single order are 5.4 Yuan and 2.5 Yuan respectively. This number is smaller than the US results estimated by Cohen et al. (2016) and Lam and Liu (2017), probably due to the lower disposable income per capita in China. Our results are unchanged in a series of robustness checks.

Our findings have important policy implications. Since 2016, the Ministry of Communications has delegated the power of supervision of online car-hailing to all city level traffic management committees. Various local regulatory policies for online car-hailing have been implemented since. This implementation has caused a sharp contraction in the scale of the online car-hailing market. The estimation of social welfare of online car-hailing suggests, other factors, such as safety and traffic problems notwithstanding, that the huge social welfare created by online car-hailing itself cannot and should not be ignored. In online car-hailing supervision, regulators should adopt policies tailor-made for local situations, and should avoid a one-size-fits-all approach to regulation.

\section{References}

Athey, S., \& Luca, M. (2019). Economists (and economics) in tech companies. Journal of Economic Perspectives, 33(1), 209-230. https://doi.org/10.1257/jep.33.1.209

Berger, T., Chen, C., \& Frey, C. B. (2018). Drivers of disruption? Estimating the Uber effect. European Economic Review, 110, 197-210. https://doi.org/10.1016/j.euroecorev.2018.05.006 
Burtch, G., Ghose, A., \& Wattal, S. (2016). Secret admirers: An empirical examination of information hiding and contribution dynamics in online crowdfunding. Information Systems Research, 27(3), 478-496. https://doi.org/10.1287/isre.2016.0642

Castillo, J. C. (2020). Who benefits from surge pricing? SSRN. https://ssrn.com/abstract=3245533

Chen, M. K., Rossi, P. E., Chevalier, J. A., \& Oehlsen, E. (2019). The value of flexible work: Evidence from Uber drivers. Journal of Political Economy, 127(6), 2735-2794. https://doi.org/10.1086/702171

Cohen, P., Hahn, R., Hall, J., Levitt, S., \& Metcalfe, R. (2016). Using big data to estimate consumer surplus: The case of Uber (No. w22627). National Bureau of Economic Research. https://doi.org/10.3386/w22627

Gao, Y., An, J., \& Quan, Y. (2016). A study on the impact of online car-booking on transportation mode selection and road traffic operation: A case study of Beijing. In Proceedings of 2016 China Urban Planning Society. Urban Transportation Planning Committee.

Ge, Y., Knittel, C. R., MacKenzie, D., \& Zoepf, S. (2020). Racial discrimination in transportation network companies. Journal of Public Economics, 190, 104205. https://doi.org/10.1016/j.jpubeco.2020.104205

Goldfarb, A., \& Tucker, C. (2019). Digital economics. Journal of Economic Literature, 57(1), 3-43. https://doi.org/10.1257/jel.20171452

Gong, J., Greenwood, B. N., \& Song, Y. (2017). Uber might buy me a Mercedes-Benz: An empirical investigation of the sharing economy and durable goods purchase. SSRN. https://doi.org/10.2139/ssrn.2971072

Grahn, R., Qian, S., Matthews, H. S., \& Hendrickson, C. (2021). Are travelers substituting between transportation network companies (TNC) and public buses? A case study in Pittsburgh. Transportation, 48(2), 977-1005. https://doi.org/10.1007/s11116-020-10081-4

Greenwood, B. N., \& Wattal, S. (2017). Show me the way to go home: An empirical investigation of ride-sharing and alcohol related motor vehicle fatalities. MIS Quarterly, 41(1), 163-187. https://doi.org/10.25300/MISQ/2017/41.1.08

Hall, J. V., \& Krueger, A. B. (2018). An analysis of the labor market for Uber's driver-partners in the United States. ILR Review, 71(3), 705-732. https://doi.org/10.1177/0019793917717222

Hall, J. D., Palsson, C., \& Price, J. (2018). Is Uber a substitute or complement for public transit? Journal of Urban Economics, 108, 36-50. https://doi.org/10.1016/j.jue.2018.09.003

Hall, J. V., Horton, J. J., \& Knoepfle, D. T. (2017). Labor market equilibration: Evidence from Uber (working paper). http://john-joseph-horton.com/papers/uber_price.pdf

Lam, C. T., \& Liu, M. (2017). Demand and consumer surplus in the on-demand economy: The case of ride sharing. Social Science Electronic Publishing, 17(8), 376-388. https://doi.org/10.2139/ssrn.2997190

Li, Z., Hong, Y., \& Zhang, Z. (2021). The empowering and competition effects of the platform-based sharing economy on the supply and demand sides of the labor market. Journal of Management Information Systems, 38(1), 140-165. https://doi.org/10.1080/07421222.2021.1870387

Liu, M., Brynjolfsson, E., \& Dowlatabadi, J. (2021). Do digital platforms reduce moral hazard? The case of Uber and taxis. Management Science, 67(8), 4643-5300. https://doi.org/10.1287/mnsc.2020.3721

Mejia, J., \& Parker, C. (2021). When transparency fails: Bias and financial incentives in ridesharing platforms. Management Science, 67(1), 166-184. https://doi.org/10.1287/mnsc.2019.3525

Moskatel, L., \& Slusky, D. (2019). Did UberX reduce ambulance volume? Health Economics, 28(7), 817-829. https://doi.org/10.1002/hec.3888

Park, J., Kim, J., Pang, M. S., \& Lee, B. (2017). Offender or guardian? An empirical analysis of ride-sharing and sexual assault (Working Paper Series 2017-006, 18-010). KAIST College of Business. https://doi.org/10.2139/ssrn.2951138 
Sadowsky, N., \& Nelson, E. (2017). The impact of ride-hailing services on public transportation use: A discontinuity regression analysis (working paper).

Shapiro, M. H. (2018). Density of demand and the benefit of Uber (working paper).

Shen, Q., \& Su, D. (2017). An empirical analysis of the impact of online car-hailing on traditional taxi industry. Journal of Henan University of Technology (Social Science Edition), (2).

Yang, H., Zhang, D., \& Sun, L. (2020). The impact of ride-hailing on traffic congestion: A complex systems perspective. Systems Engineering, 38(3), 8.

Yu, H., Tian, L., Jiang, G., \& Chen, Y. (2018). Sharing economy: Theoretical construction and research progress. Nankai Management Review, (21).

Zhang, Z., \& Li, B. (2017). A quasi-experimental estimate of the impact of p2p transportation platforms on urban consumer patterns. In Proceedings of the 23rd ACM SIGKDD International Conference on Knowledge Discovery and Data Mining (pp. 1683-1692). https://doi.org/10.1145/3097983.3098058

Zoepf, S. M., Chen, S., Adu, P., \& Pozo, G. (2018). The economics of ride-hailing: Driver revenue, expenses and taxes (CEEPR Working paper 5). Massachusetts Institute of Technology. 